\title{
Microvascular Dysfunction, Myocardial Ischemia, and Progression to Heart Failure in Patients with Hypertrophic Cardiomyopathy
}

\author{
Franco Cecchi - Aurelio Sgalambro - Massimo Baldi • \\ Barbara Sotgia - Davide Antoniucci - Paolo G. Camici • \\ Roberto Sciagrà • Iacopo Olivotto
}

Received: 14 September 2009 / Accepted: 5 October 2009 /Published online: 3 November 2009

(C) Springer Science + Business Media, LLC 2009

\begin{abstract}
Microvascular dysfunction can be demonstrated in most patients with hypertrophic cardiomyopathy (HCM), both in the hypertrophied and nonhypertrophied myocardial walls, mostly due to intimal and medial hyperplasia of the intramural coronary arteries and subsequent lumen reduction. As a consequence, regional myocardial ischemia may be triggered by exercise, increased heart rate, or arrhythmias, in areas which are unable to increase myocardial blood flow. In patients with HCM, microvascular dysfunction leading to severe myocardial hypoperfusion during maximal hyperemia represents a strong predictor of
\end{abstract}

F. Cecchi $\cdot$ A. Sgalambro $\cdot$ M. Baldi $\cdot$ I. Olivotto

Regional Referral Center for Myocardial Diseases,

Department of Cardiology,

Azienda Ospedaliera Universitaria Careggi,

Florence, Italy

P. G. Camici

Medical Research Council Clinical Sciences Center,

Hammersmith Hospital, Imperial College,

London, UK

F. Cecchi $(\bowtie)$

Dipartimento Cuore e Vasi-S. Luca vecchio,

Azienda Ospedaliera Universitaria Careggi,

Viale Pieraccini 17, 50139,

50129 Florence, Italy

e-mail: cecchif@aou-careggi.toscana.it

B. Sotgia $\cdot$ R. Sciagrà

Department of Nuclear Medicine,

Azienda Ospedaliera Universitaria Careggi,

Florence, Italy

D. Antoniucci

Department of Cardiology, Invasive Cardiology

Azienda Ospedaliera Universitaria Careggi,

Florence, Italy unfavorable outcome, left ventricular remodeling with progressive wall thinning, left ventricular dysfunction, and heart failure. Accurate quantitative assessment of microvascular dysfunction and myocardial ischemia is not easily feasible in clinical practice. Although signs of inducible myocardial ischemia may be detected by electrocardiogram, echocardiography, or myocardial scintigraphy, the vasodilator response to dipyridamole by positron emission tomography is considered the method of choice for the assessment of maximal regional and global flow. Cardiac magnetic resonance provides further information, by late gadolinium enhancement (LGE), which may show areas where replacement fibrosis has occurred following microvascular ischemia and focal necrosis. LGE areas colocalize with severe regional microvascular dysfunction, are associated with increased prevalence of ventricular arrhythmias, and show more extensive distribution in the late stages of the disease, when heart failure is the dominant feature. The present review aims to provide a concise overview of the available evidence of microvascular dysfunction and ischemia eventually leading to disease progression and heart failure in HCM patients.

Keywords Hypertrophic Cardiomyopathy · Microvascular Dysfunction · Myocardial Ischemia $\cdot$ Positron Emission Tomography . Outcome

Hypertrophic cardiomyopathy (HCM), the most common genetically determined heart disease, is characterized by a wide range of clinical manifestations, from sudden and unexpected cardiac death to a progressive clinical course with functional limitation and heart-failure-related complications such as atrial fibrillation (AF) and stroke, ultimately 
leading to heart-failure-related death $[9,18,25,30,48]$. Myocardial ischemia occurring at the microvascular level is a major determinant of the clinical outcome in HCM patients and is held responsible for some of the adverse manifestations of the disease ranging from myocardial infarction to left ventricular (LV) remodeling, systolic dysfunction, ventricular arrhythmias, and even sudden death $[2,3,33,42]$. However, the assessment of ischemia in HCM patients is limited in clinical practice and is not yet incorporated in the HCM management guidelines. This is an important limitation to the understanding of the disease pathophysiology and risk assessment in the individual patient. However, recent advances in imaging technology now provide a more comprehensive perspective in the evaluation of the causes and consequences of ischemia at the myocardial level. Here, we review the existing evidence for microvascular dysfunction and ischemia and its causes in HCM, as well as the impact it may have in the clinical progression to systolic dysfunction and heart failure, including the "end-stage phase."

\section{The Substrate: Myocardial Ischemia and Infarction Due to Small Vessel Disease}

In 1976, Bartoloni et al. described a case of acute myocardial infarction leading to rapidly progressing heart failure and death in an 8-year-old child with HCM, who was playing football [2]. Postmortem examination of the heart revealed massive asymmetrical hypertrophy of the interventricular septum and evidence of recent extensive anteroseptal necrosis in the area of a deeply tunneled left anterior descending coronary artery [3]. At the microscopic level, disarray of the myocardial fibers, areas of fibrosis, and marked structural abnormalities of the intramural

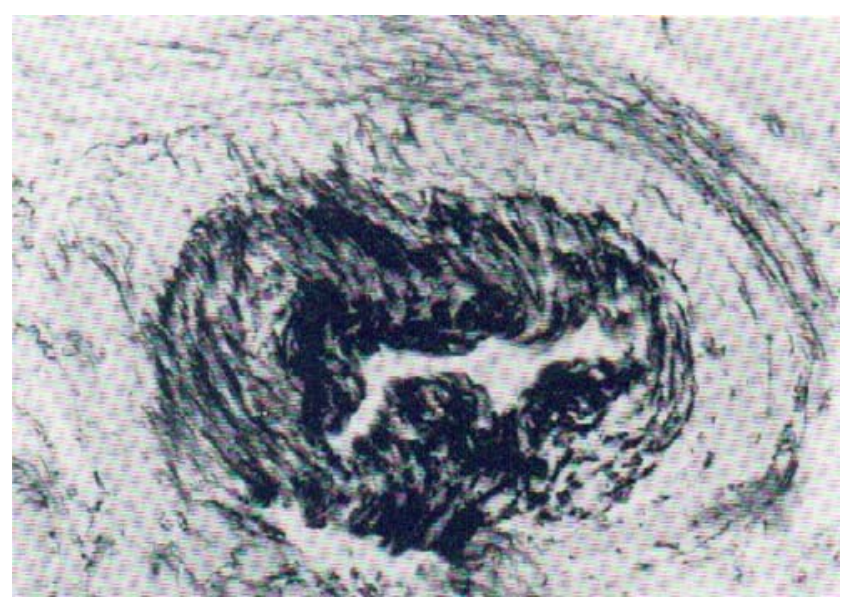

Fig. 1 Microvascular remodeling in HCM. Abnormal intramural coronary artery in a hypertrophied and extensively fibrosed region of the ventricular septum. From Bartoloni Sant Omer [2] coronary arteries were noted. The latter were characterized by intima-medial thickening, causing deformation and severe narrowing of the vessel lumen (Fig. 1). The authors concluded that these vessel abnormalities justified a severe chronic impairment of myocardial perfusion within the interventricular septum, potentially exposing the patient to repetitive ischemia and ultimately to myocardial infarction. Such observation represents the earliest report describing myocardial infarction in the context of small vessel disease and tunneled left anterior descending in HCM. Subsequently, other postmortem studies on patients who died suddenly showed frequent and often extensive areas of myocardial necrosis, in the absence of atherosclerotic coronary artery disease, associated with all stages of ischemic injury, including a chronic-phase characterized by postnecrotic replacement-type fibrosis [26, 33, 3]. Anatomical remodeling of the intramyocardial coronary arterioles, characterized by thickening of the vessel wall due to medial hypertrophy and/or intimal hyperplasia, and perimysial and intra-arterial fibrosis were associated with severely decreased luminal area in the majority of cases. These features may be observed both in the hypertrophied and nonhypertrophied myocardial segments, with considerable regional variation within the LV [33], and are commonly detected in surgical specimens obtained in patients undergoing myectomy (Fig. 2). The genesis of the small vessel abnormalities observed in patients with HCM, which have not been consistently reproduced in transgenic models of the disease, still lacks a definite explanation.

In addition to the intrinsic structural remodeling of the microcirculation, other potential relevant factors include reduced capillary density, myocyte disarray, interstitial fibrosis, and increased oxygen demand of the hypertrophied

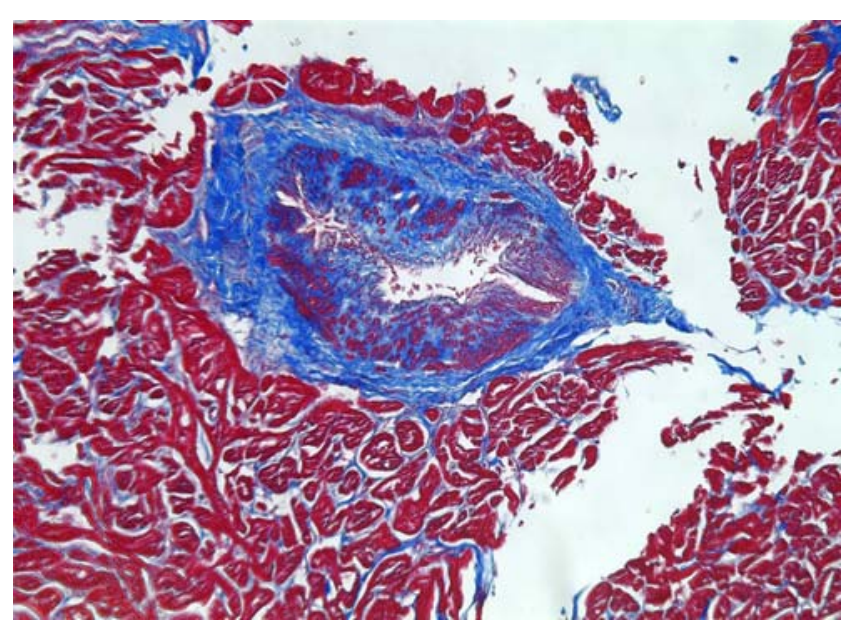

Fig. 2 Intramural coronary artery with medial hypertrophy and fibrosis, perimysial fibrosis, and markedly reduced lumen. The specimen was obtained after extended myectomy performed in an 18-year-old HCM patient with an MYH7 missense mutation (trichromic stain, $\times 10$ ) 


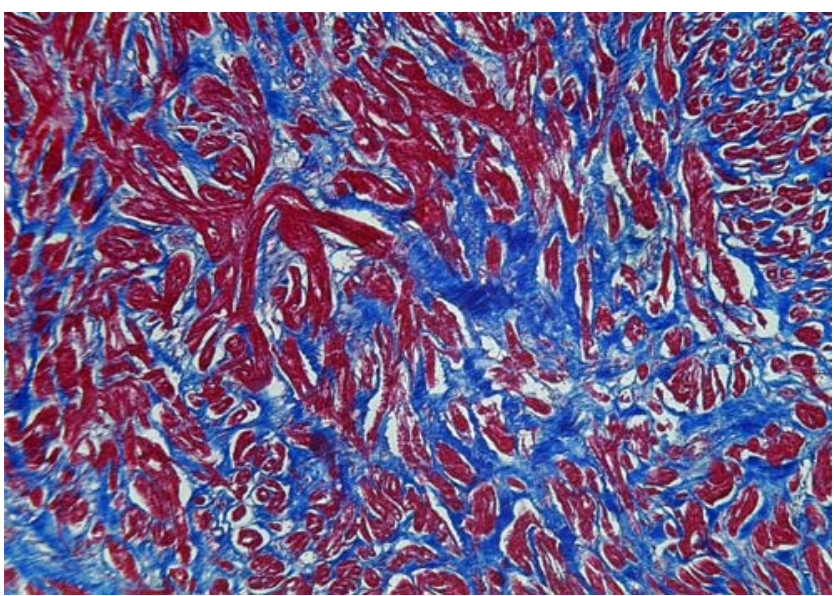

Fig. 3 Diffuse myocardial disarray and interstitial fibrosis (in blue) in the same patient (trichromic stain, $\times 10$ )

cardiomyocytes [19-21]. Such abnormalities constitute the anatomical basis for microvascular dysfunction and represent the substrate for ischemia in HCM patients. Regional ischemia may develop when myocardial oxygen demand is increased, ultimately leading to myocyte death and replacement fibrosis (Fig. 3). In the long term, cardiomyocyte loss and generation of fibrosis, in association with the primary genetically determined myocyte functional abnormalities, may result in disease progression characterized by adverse LV remodeling, progressive wall thinning, and dysfunction $[32,39]$.

\section{Clinical Features}

Most HCM patients complain of atypical chest pain or tightness occurring at rest or during exercise. Typical angina is rarely reported and should be carefully evaluated. Coronary arteriography should be considered, in order to exclude causes of ischemia involving the epicardial coronary circulation, such as atherosclerotic disease in the adults, myocardial tunneling, or anomalous origin of coronary arteries in the young. However, myocardial hypoperfusion and ischemia may be clinically silent, as reflected by HCM patients with severe regional hypoperfusion after dipyridamole injection and even after alcohol injection in the course of septal alcohol ablation procedures $[4,11,22]$. Occasionally, patients complain of angina during supraventricular arrhythmias with rapid ventricular response, and anecdotal reports clearly show the occurrence of transmural ischemia, with ST segment elevation, during paroxysmal AF, occasionally leading to ventricular fibrillation (Fig. 4) [47]. Less frequently, ischemia may be associated with frequent ventricular ectopics or recurrent runs of nonsustained ventricular tachycardia on ambulatory electrocardiogram (ECG).

\section{Assessment of Myocardial Ischemia}

Over the last decades, several techniques have been employed to show the occurrence of myocardial ischemia in patients with HCM but all share significant limitations (Table 1). Ischemic ST-T changes on the electrocardiogram have been documented during ambulatory ECG (Fig. 5), exercise testing, rapid atrial pacing, AF with rapid ventricular response rate [14, 42, 47], and stress echocardiography with dipyridamole [22]. After alcohol is injected in the first or second septal artery for septal ablation, most HCM patients show typical ST elevation in anterior leads, but in some patients minor ECG changes may be observed [11]. Unfortunately, the ECG is neither a sensitive nor a specific marker of ischemia, due to marked basal ST-T alterations secondary to LV hypertrophy in most patients [14, 34]. Often, LV wall motion abnormalities may be detected by stress $2 \mathrm{D}$ echocardiography during dipyridamole or dobutamine infusion. These may be suggestive of myocardial ischemia, but their detection is not easy and their clinical significance remains unclear [24].

Conventional scintigraphic techniques have been repeatedly used in HCM patients $[5,12,13]$. Defects during single-photon emission computed tomography (SPECT) thallium-201 myocardial perfusion imaging are common. Fixed defects are associated with increased LV cavity dimensions and reduced systolic function and are usually interpreted as areas of scarring. Reversible defects induced by exercise have been interpreted as markers of myocardial ischemia because of a high concordance with metabolic evidence of ischemia. Unfortunately, they are not able to provide absolute quantification of myocardial flow and fail to correlate with symptoms and outcome, due to intrinsic limitations of the technique [12].

Positron emission tomography (PET) allows noninvasive assessment of myocardial blood flow (MBF) in patients with LV hypertrophy and HCM, both at rest and in conditions of maximal vasodilatation induced by dipyridamole infusion, by injection of tracers such as ${ }^{13} \mathrm{~N}$-labeled ammonia or ${ }^{15} \mathrm{O}$-labeled water $[4,8,23,45$, 46]. However, it must be emphasized that dipyridamoleinduced maximal vasodilation is not equivalent to physiologic exercise, which, unfortunately, cannot be used with PET, as PET requires relative absence of movement during the study. Recent advances in technology greatly improved spatial resolution, which is well superior to that of SPECT and allowed PET noninvasive quantification of regional MBF. In the majority of HCM patients studied with PET, while resting $\mathrm{MBF}$ is not dissimilar from that of normal controls, increase in MBF after dipyridamole infusion is significantly reduced, even below resting MBF, suggest- 

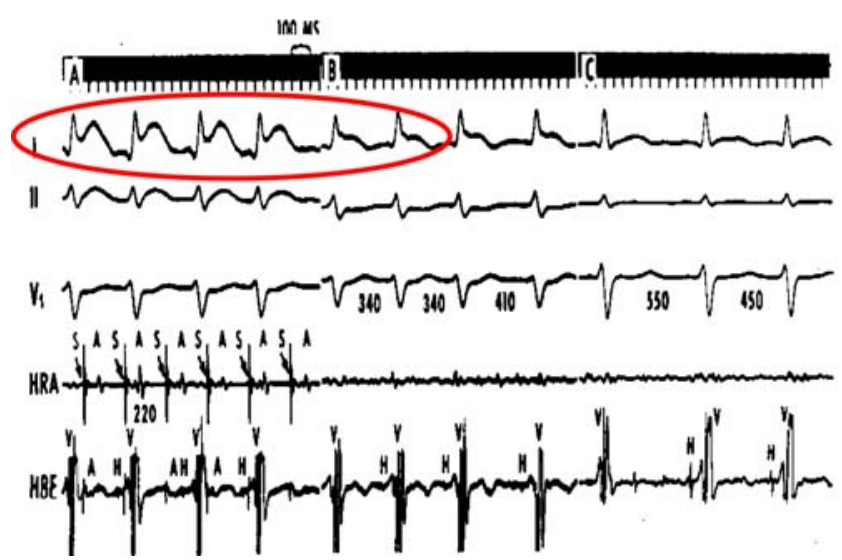

Fig. 4 Atrial fibrillation inducing myocardial ischemia and VF in a young patient (27 years old) successfully resuscitated from a cardiac arrest. During EP study, atrial pacing induced AF with rapid AV

ing absolute hypoperfusion (Fig. 6) [4]. On average, after dipyridamole injection, HCM patients are able to increase their global mean MBF less than twofold, and sometimes as low as 1 or even less, compared to an average fourfold in healthy human volunteers. The reduction of coronary

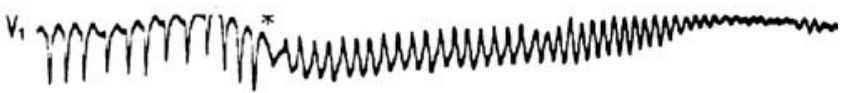
HRA

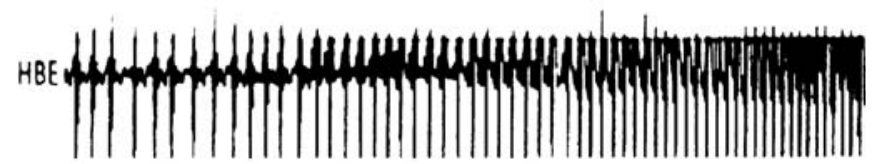

conduction; acute ischemia with ST elevation occurred, followed by ventricular fibrillation. Reproduced with permission of the American College of Cardiology; from [47]

artery reserve, which is the ratio between the maximal and basal MBF, is independent of age and maximal wall thickness [4, 23]. It must also be emphasized that MBF impairment is characterized by marked regional and individual variability. Thus, each patient may exhibit

Table 1 Clinical relevance and limitations of different techniques for the assessment of microvascular dysfunction and ischemia in HCM. Reproduced from Maron et al. [29]

\begin{tabular}{|c|c|c|c|}
\hline Method & Finding & Clinical Relevance & Limitations \\
\hline Clinical history & Nonanginal chest pain & Ongoing microvascular ischemia & $\begin{array}{l}\text { Poor sensitivity and specificity. Should prompt } \\
\text { investigation for alternative causes if severe (CAD, } \\
\text { bridging) }\end{array}$ \\
\hline Troponin I & Increased serum levels & Acute myocyte injury & $\begin{array}{l}\text { Limited utility in HCM due to frequent lack of symptom } \\
\text { during ischemia. If markedly elevated in association with } \\
\text { symptoms, should also prompt exclusion of CAD }\end{array}$ \\
\hline Holter ECG & ST-T changes & Ongoing ischemia & $\begin{array}{l}\text { Poor sensitivity; baseline ECG abnormalities often limit } \\
\text { reliability }\end{array}$ \\
\hline Exercise testing & $\begin{array}{l}\text { ST-T changes/ } \\
\text { symptoms }\end{array}$ & Inducible ischemia & $\begin{array}{l}\text { Poor sensitivity; baseline ECG abnormalities often limit } \\
\text { reliability }\end{array}$ \\
\hline $\begin{array}{l}\text { Stress } \\
\text { echocardiography }\end{array}$ & $\begin{array}{l}\text { Regional wall motion } \\
\text { abnormalities }\end{array}$ & Inducible ischemia & Limited experience in HCM; likely poorly sensitive \\
\hline SPECT & $\begin{array}{l}\text { Regional perfusion } \\
\text { abnormalities }\end{array}$ & $\begin{array}{l}\text { Regional microvascular dysfunction, } \\
\text { may predict end-stage progression }\end{array}$ & Lack of MBF quantitation; suboptimal sensitive \\
\hline $\begin{array}{l}\text { Coronary } \\
\text { angiography }\end{array}$ & $\begin{array}{l}\text { Myocardial bridging/ } \\
\text { tunneling of LAD, } \\
\text { associated CAD }\end{array}$ & $\begin{array}{l}\text { Exclusion of associated causes of } \\
\text { ischemia }\end{array}$ & Not practical for assessment of microvascular function \\
\hline $\begin{array}{l}\text { Coronary lactate } \\
\text { (serum) levels }\end{array}$ & Lactate production & Anaerobic metabolism/ischemia & Invasive, not applicable in routine clinical practice \\
\hline PET & $\begin{array}{l}\text { Reduced coronary } \\
\text { reserve/blunted } \\
\text { maximal MBF }\end{array}$ & $\begin{array}{l}\text { Extent and distribution of } \\
\text { microvascular dysfunction, predicts } \\
\text { outcome and LV remodeling }\end{array}$ & Limited availability and expensive. Radiation exposure \\
\hline $\begin{array}{l}\text { CMR stress } \\
\text { perfusion }\end{array}$ & $\begin{array}{l}\text { Reduced coronary } \\
\text { reserve/blunted } \\
\text { maximal MBF }\end{array}$ & $\begin{array}{l}\text { Extent and distribution of } \\
\text { microvascular dysfunction }\end{array}$ & $\begin{array}{l}\text { Still not widely available; time consuming/expensive. } \\
\text { Validation less robust than PET }\end{array}$ \\
\hline $\begin{array}{l}\text { CMR late } \\
\text { gadolinium }\end{array}$ & $\begin{array}{l}\text { Delayed contrast } \\
\text { enhancement }\end{array}$ & $\begin{array}{l}\text { Replacement scarring after recurrent } \\
\text { ischemia }\end{array}$ & Prognostic significance still under investigation \\
\hline
\end{tabular}

$C A D$ coronary artery disease, $C M R$ cardiovascular magnetic resonance, $E C G$ electrocardiogram, $H C M$ hypertrophic cardiomyopathy, $L A D$ left anterior descending coronary artery, $L V$ left ventricular, $M B F$ myocardial blood flow, PET positron emission tomography, SPECT single-photon emission computed tomography 
Fig. 5 ECG ambulatory monitoring recording at rest (a) and during mild effort (walking; b) in a 48-year-old woman with HCM. During activity, marked ST-T segment downsloping appears, associated with angina and mild dyspnea a

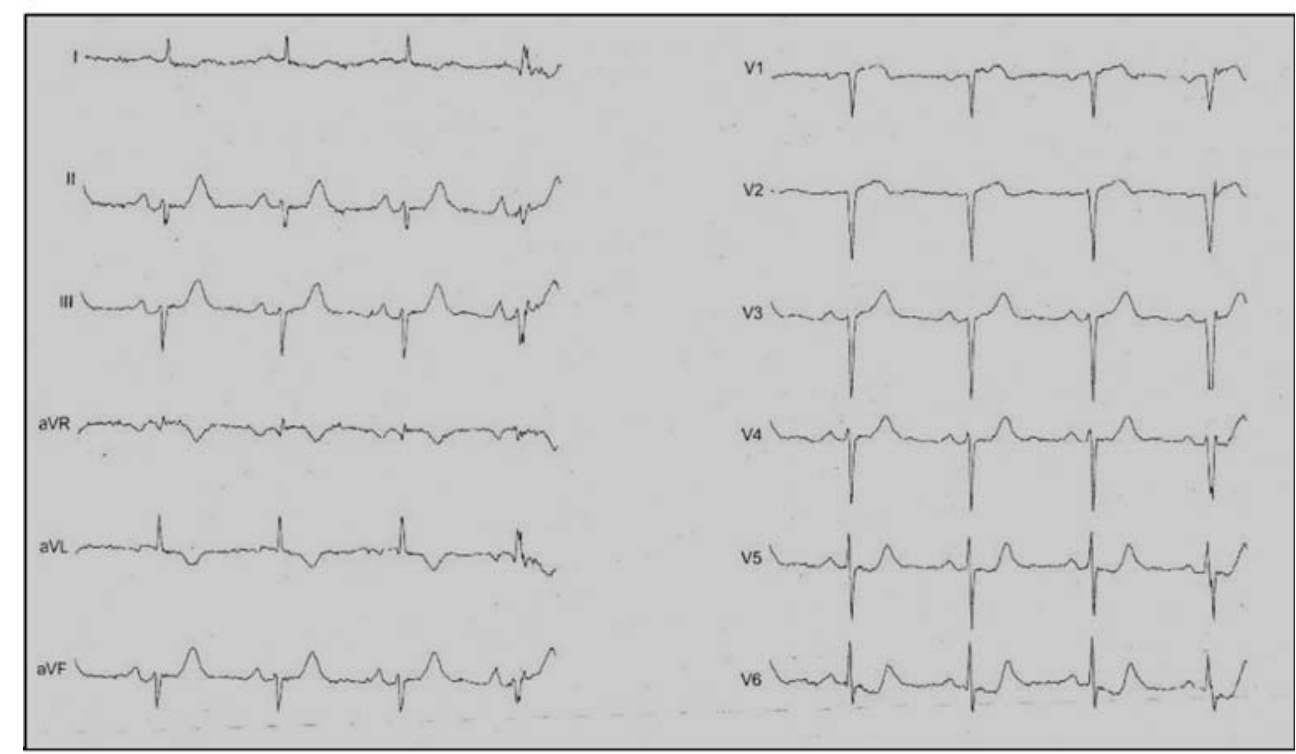

b

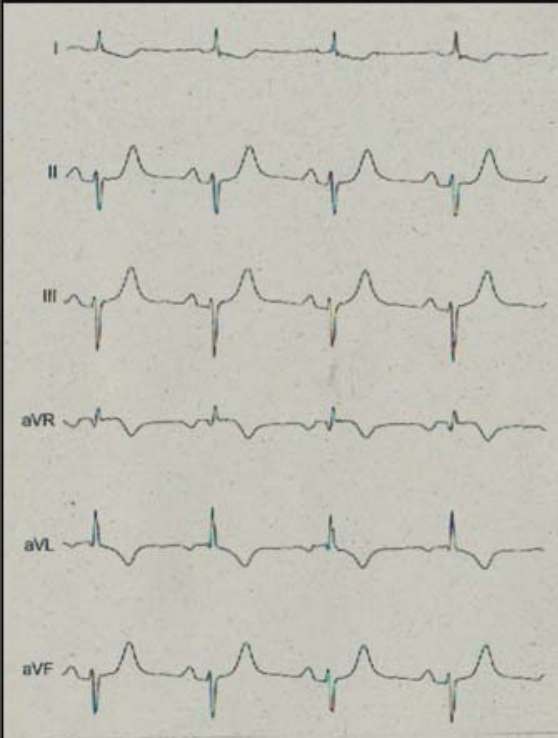

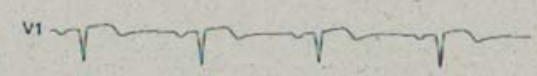
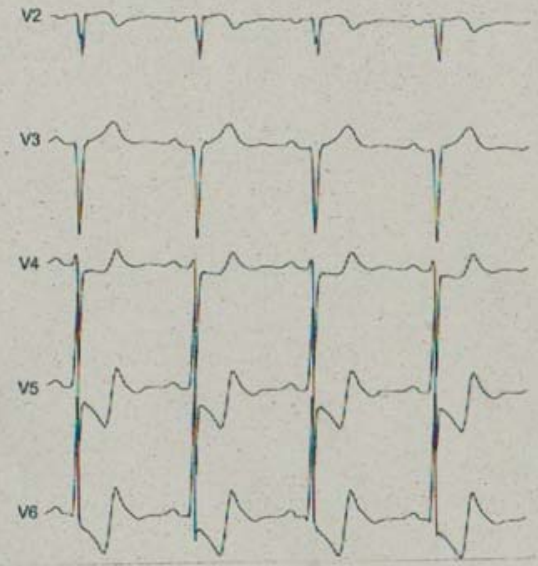

severe impairment of MBF in certain areas of the myocardium, adjacent to other in which perfusion may be somehow preserved. Moreover, in agreement with the diffuse abnormalities of the intramural coronary arterioles described by pathologists, dipyridamole MBF may be markedly impaired not only in the hypertrophied ventricular septum, but also in the nonhypertrophied or less thickened free wall of the left ventricle [4, 17].

Cardiac magnetic resonance (CMR), besides representing a gold standard for morphological assessment and increasing the diagnostic sensitivity, can now provide quantification on myocardial flow [35, 43], although it is still time consuming and not available in clinical practice. CMR, however, provides further insight into the effects of regional hypoperfusion and ischemia in HCM patients, by late gadolinium enhancement (LGE), which is believed to reflect areas of edema or fibrosis resulting from recurrent microvascular ischemia and is associated with an increased incidence of arrhythmias [1].

In a recent study by our group, flow impairment was on average more severe in $\mathrm{LV}$ regions with or adjacent to areas of LGE [46], suggesting a causal role of microvascular dysfunction in producing ischemia and replacement fibrosis, as originally postulated by some authors [33]. LGE was shown to colocalize with microvascular dysfunction. In segments without LGE but contiguous to fibrotic areas, maximum flow following dipyridamole infusion was significantly lower than in those remote from LGE. Although there is no strong evidence that local microvascular dysfunction is responsible for the occurrence of focal 
Fig. 6 Polar map of regional quantitative myocardial perfusion after dipyridamole injection by PET in the same patient as in Figs. 2 and 3. As shown, maximal MBF ranged from 0.82 to $2.68 \mathrm{ml} / \mathrm{mg}$ per minute. The color scale shows highest values of flow in yellow and lowest in dark red

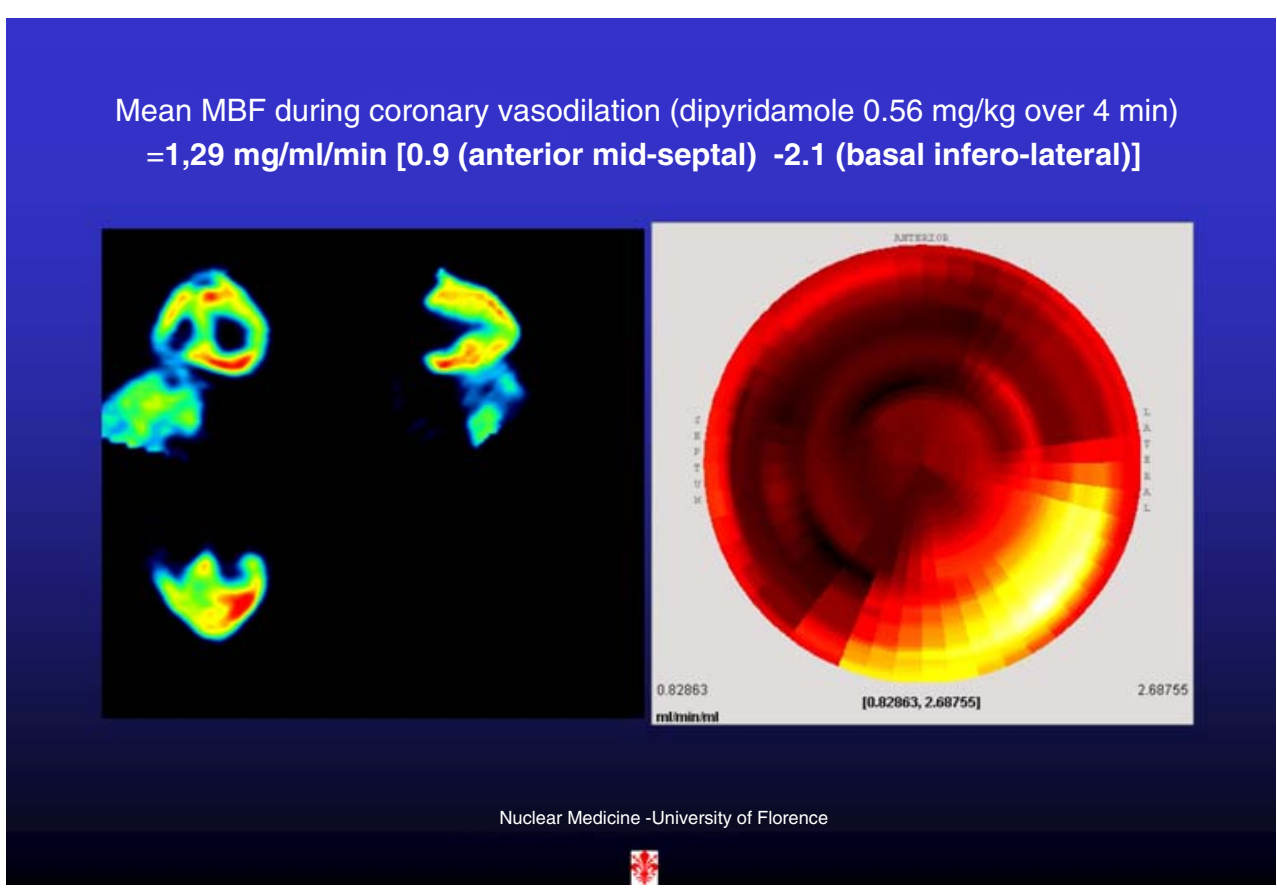

necrosis and replacement fibrosis, considerable indirect evidence that increasing degrees of coronary microvascular dysfunction might play a causative role for myocardial fibrosis in patients with HCM supports this hypothesis (Fig. 7) [46].

\section{The Triggers: Exercise, Sinus Tachycardia, Supraventricular Arrhythmias, LV Intraventricular Obstruction}

The documentation of microvascular dysfunction is not per se equivalent to demonstrating myocardial ischemia [29]. However, an impaired capacity to increase flow on demand at the microvascular level sets the stage for recurrent myocardial ischemia, whenever myocardial oxygen requirements exceed regional blood flow. The most important and common triggers acting upon the substrate of microvascular dysfunction to produce ischemia in HCM are represented by sinus tachycardia or increased ventricular rate, which may occur during physical exercise, supraventricular arrhythmias such as paroxysmal AF, and other specific or nonspecific conditions increasing oxygen consumption, such as dynamic LV intraventricular obstruction, anemia, uncontrolled arterial hypertension, and hyperthyroidism

In patients with HCM, coronary sinus lactate production is greatly increased during atrial pacing as a consequence of myocardial ischemia, sometimes associated with angina. Moreover, coronary sinus blood taken after dipyridamole infusion showed a significant $\mathrm{Ph}$ reduction, which was interpreted as a sign of dipyridamole-induced myocardial ischemia [14]. Sports involving intense effort and anaerobic isometric activities resulting in rapid heart rate acceleration are expected to bear the most adverse ischemic consequences, also due to a critical reduction in the diastolic time critical for myocardial perfusion. These considerations therefore provide a rationale for exercise limitation and pharmacological control of maximal heart rates in young active patients, in order to prevent asymptomatic myocardial ischemia.

Supraventricular arrhythmias and in particular AF are the most common complication of $\mathrm{HCM}$, occurring in about $20-25 \%$ of patients in a 10 -year follow-up $[7,38]$. The onset of paroxysmal AF is almost never well tolerated by HCM patients, unless they are on active treatment with atrioventricular blocking agents, such as diltiazem, verapamil, or $\beta$-blockers. In some patients, the combination of loss of atrial contraction and rapid ventricular response may trigger a vicious circle of low cardiac output, regional hypoperfusion, and acute myocardial ischemia, which may generate malignant ventricular arrhythmias (Fig. 4) [47]. To a less dramatic extent, patients with permanent AF may also be exposed to recurrent ischemia in the presence of a significantly impaired coronary flow reserve, if their ventricular rate is not adequately controlled in their daily activities. Moreover, MBF is significantly reduced in patients with at least one episode of atrial fibrillation, suggesting an association and possibly a causal role for microvascular dysfunction and myocardial hypoperfusion in determining the arrhythmia [45].

Finally, in the presence of resting or provocable dynamic LV outflow obstruction, observed in more than two thirds of HCM patients [31], the pathological increase in intracavitary pressures may substantially contribute to myocar- 

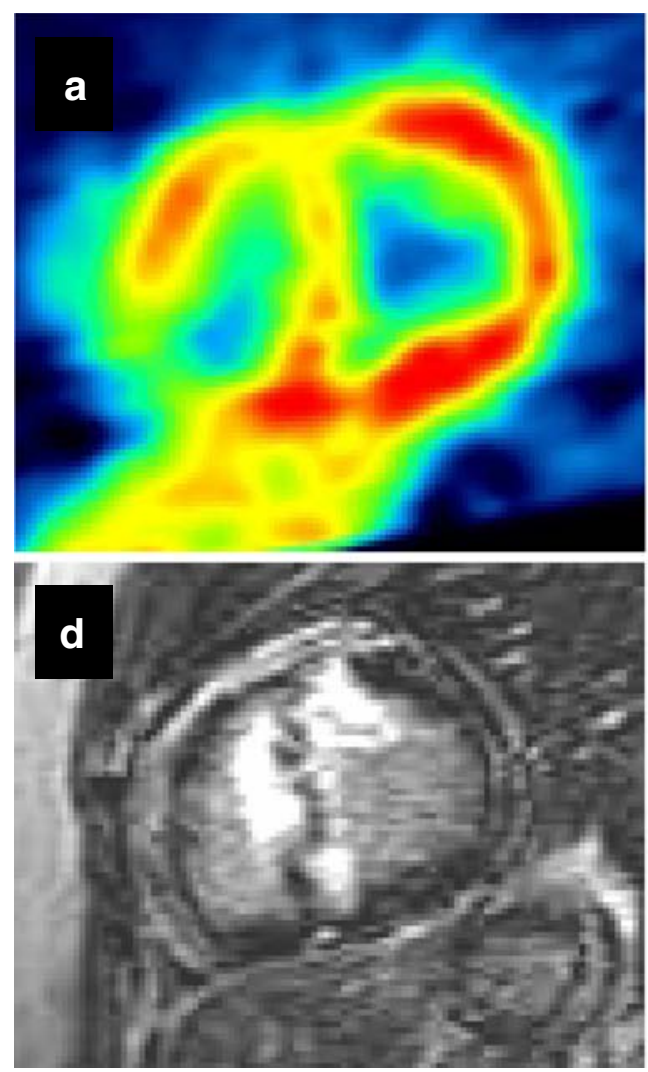

Fig. 7 Relationship of dipyridamole MBF to delayed contrast enhancement (DCE) in a 28-year-old female patient with HCM. a $\mathrm{NH}_{3}$ PET short-axis slice at the level of the basal level LV segments. The color scale shows highest values of flow in red and lowest in green. b First pass magnetic resonance short-axis slice at the base of the LV, showing diffuse septal hypertrophy. c Following gadolinium infusion, extensive DCE is evident (white signal), involving the interventricular septum and extending into the anterior wall. There is

dial hypoperfusion at the subendocardial level and adverse outcome $[20,27,28]$. Such consequences appear to be particularly adverse in patients with midventricular obstruction, in whom progressive apical dilatation may occur with scar formation, which may further progress to LV dilatation, dysfunction, and heart failure [10, 16]. Therefore, although related to a number of other pathophysiological effects, the remarkable clinical benefits of surgical myectomy may be partly mediated by a reduction of microvascular dysfunction and ischemia at the subendocardial level $[6,40]$.

\section{Disease Progression to Heart Failure and the End-Stage Phase as a Consequence of Ischemia}

Severe microvascular dysfunction has an impact on longterm outcome in $\mathrm{HCM}$ patients. In a cohort of $51 \mathrm{HCM}$ patients prospectively followed for more than 8 years after a PET flow study, a dipyridamole MBF value in the lowest tertile $(<1.11 \mathrm{mg} / \mathrm{ml}$ per minute) was the most powerful independent predictor of cardiovascular mortality, after
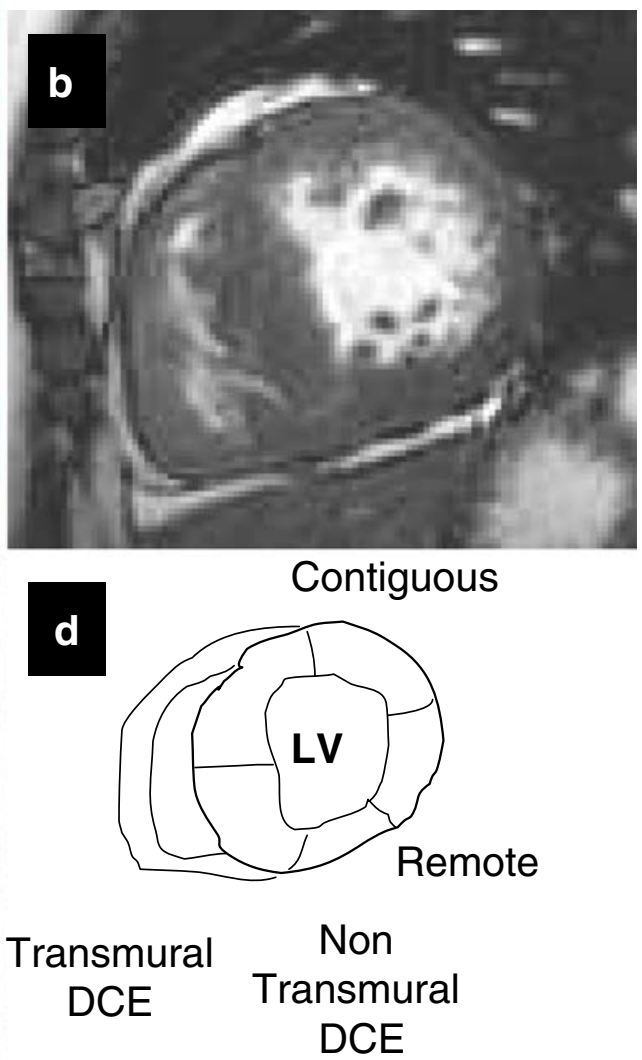

close agreement between DCE and areas of reduced flow at PET. d Diagram illustrating the nomenclature employed for the classification of myocardial segments with regard to the extent and proximity of DCE, as follows: (1) transmural DCE; (2) nontransmural DCE; (3) without DCE but contiguous to the DCE segments; (4) without DCE and remote from DCE; numbers in italic indicate $\mathrm{hMBF}(\mathrm{ml} / \mathrm{min}$ per gram) in the segment. Reproduced with permission from [46]

adjustment for other clinically relevant variables. Relative risk for the composite end point of death, stroke, or progression to New York Heart Association class III-IV was almost 20-fold in the lowest tertile of flow (Fig. 8) [8]. Of note, at the time of PET, only few of these patients would have been considered at high risk based on the current guidelines [27, 28], as severe microvascular dysfunction could be demonstrated several years before their clinical progression [8]. Furthermore, patients with the most impaired microvascular response to dipyridamole showed a significantly higher incidence of long-term adverse LV remodeling, with chamber dilatation, wall thinning, and decline in systolic function (Fig. 9) [39]. All patients in this cohort who subsequently developed severe heart failure symptoms and progressed to the end stage originally belonged to the lowest tertile of dipyridamole MBF. Therefore, severe microvascular dysfunction seems to be a critical risk factor for systolic dysfunction, disease progression, and heart failure in HCM patients, and assessment of maximal MBF after dipyridamole injection represents a valuable tool for the identification of patients at higher risk. 
Fig. 8 Microvascular dysfunction is associated with adverse outcome. Evidence that dipyridamole MBF by PET predicts adverse disease outcome in patients with HCM. Cardiovascular mortality was greatest in HCM patients with a dipyridamole MBF value in the lowest tertile. Reproduced with permission of Massachusetts Medical Society; from [8] a

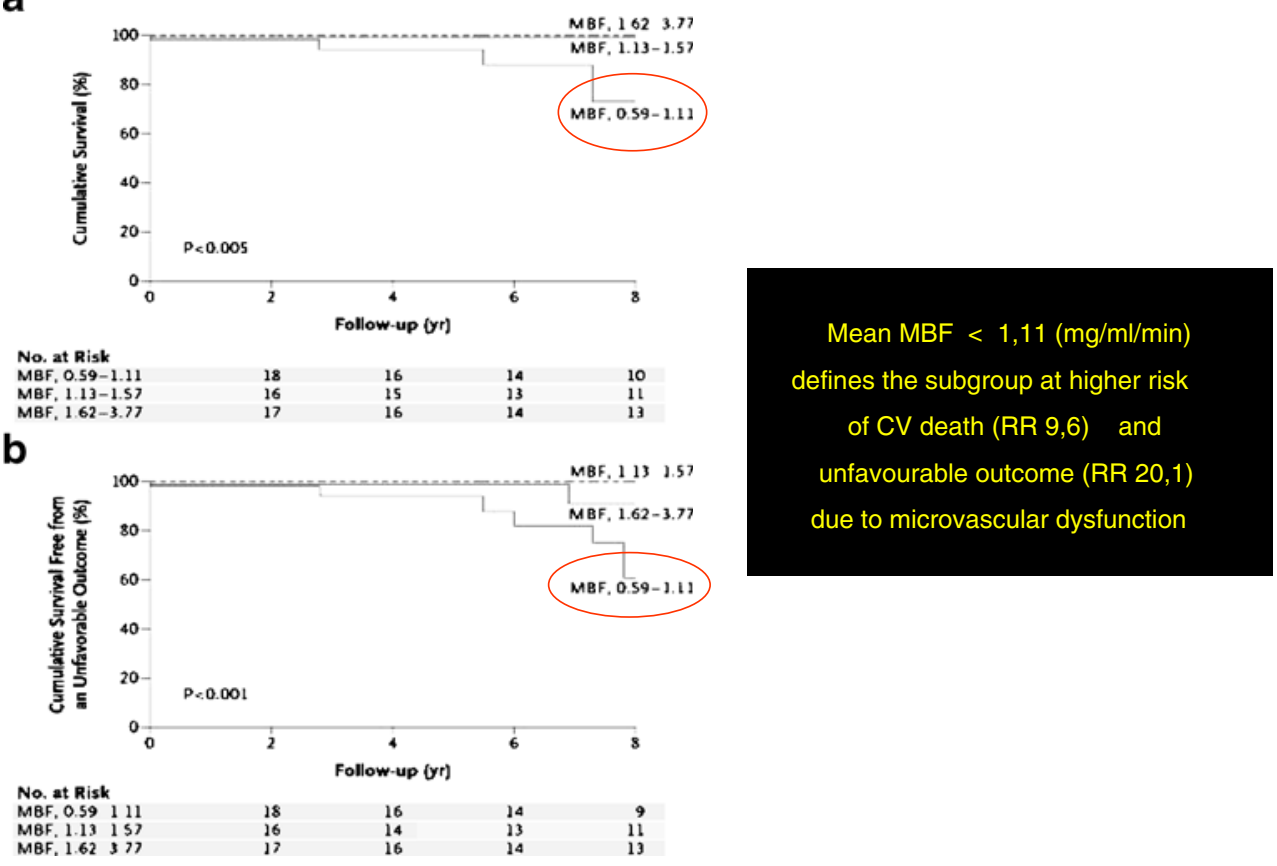

\section{Management of Microvascular Dysfunction}

At present, no specific treatment has been shown to significantly improve impaired microvascular function. In clinical practice, control of potential triggers of ischemia still represents the most effective strategy. Avoidance of intense competitive sports and strenuous physical effort, control of heart rate by AV node blocking agents, relief of

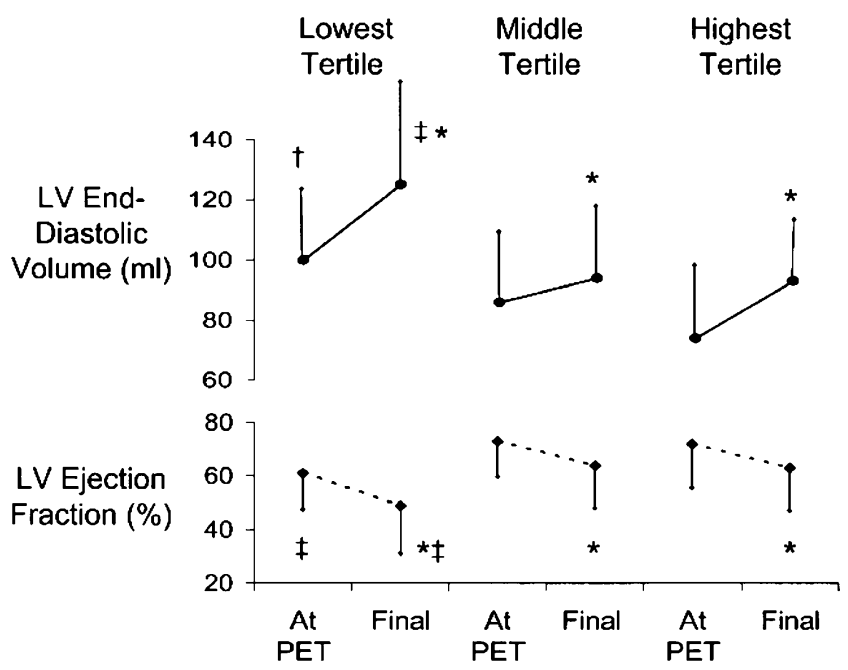

Fig. 9 Microvascular dysfunction and LV remodeling. Comparison of LV end diastolic dimension and ejection fraction at the time of PET study and at final evaluation expressed as tertiles of MBF after dipyridamole infusion. Patients with a dipyridamole MBF value in the lowest tertile showed the greatest change in cavity size and ejection fraction. Vertical bars indicate mean \pm SD for each group. Symbols: $*=p<0.05$ versus same group at the time of PET scan; $\dagger=p<0.05$ versus patients in the highest tertile; $t=p<0.05$ versus patients in other tertiles. Reproduced with permission [39]
LV outflow obstruction, and management of comorbidity such as anemia, thyroid dysfunction, and hypertension are all likely to be beneficial, although no randomized studies are available for evidence-based implementation. Diltiazem, verapamil, and $\beta$-blockers are known to improve symptoms $[25,44]$, as in patients with coronary artery disease, probably via a reduction in heart rate and oxygen consumption. Treatment with verapamil did not show improvement in MBF and coronary flow reserve in HCM patients, although it led to improved redistribution of transmural $\mathrm{MBF}$ and increased subendocardial perfusion [17]. Recent studies of the no-reflow phenomenon following myocardial infarction have confirmed a clinically relevant vasodilator capacity of verapamil on the coronary microcirculation [41]. In patients at risk of developing atrial fibrillation, the prophylactic use of amiodarone may improve symptoms and also prevent arrhythmic recurrences. Angiotensin-converting enzyme (ACE) inhibitors have also been shown to improve transmural myocardial perfusion and restore impaired subendocardial flow in a canine model of dilated cardiomyopathy, by virtue of a nitricoxide-dependent mechanism [37]. In a small pilot study, ACE inhibitors have been shown to reverse small vessel changes, improve endothelial function, and reduce periarteriolar fibrosis in hypertensive patients [36]. Spironolactone is also a potentially useful drug in this context, for its capability of interacting with the renin-angiotensin system, reducing both coronary microvascular remodeling and fibrosis [29]. Recently, nebivolol has been shown to significantly improve microvascular function in patients with idiopathic dilated cardiomyopathy [15]. Therefore, pharmacological treatment may in fact improve the status of the 
coronary microcirculation in HCM. However, further research is much needed in this field, leading to the development of novel agents truly capable of reversing microvascular dysfunction and its long-term consequences in HCM patients.

\section{Conclusions}

Microvascular dysfunction is an important feature in patients with HCM, although it is rarely considered in clinical practice. Its assessment, through the value of maximal blood flow after dipyridamole injection by PET, predicts long-term clinical outcome and LV remodeling. Myocardial ischemia may develop in underperfused myocardial areas and contribute to the development of the "dilated or end-stage phase." The clinical armamentarium to improve MBF is currently limited, although novel drugs are expected in the near future.

\section{References}

1. Adabag, A. S., Maron, B. J., Appelbaum, E., Harrigan, C. J., Buros, J. L., Gibson, C. M., et al. (2008). Occurrence and frequency of arrhythmias in hypertrophic cardiomyopathy in relation to delayed enhancement on cardiovascular magnetic resonance. Journal of the American College of Cardiology, 51, 1369-1374.

2. Bartoloni Saint Omer, F., Gori, F., Marchi, F., \& Pagnini, P. (1976). Infarto miocardico acuto settale in bambino di otto anni con miocardiopatia ipertrofica ostruttiva. Archivio "De Vecchi", LXI, 41-54

3. Basso, C., Thiene, G., Corrado, D., et al. (2000). Hypertrophic cardiomyopathy and sudden death in the young: pathologic evidence of myocardial ischemia. Human Pathology, 31, 988-98.

4. Camici, P. G., Chiriatti, G., Lorenzoni, R., et al. (1991). Coronary vasodilation is impaired in both hypertrophied and nonhypertrophied myocardium of patients with hypertrophic cardiomyopathy: A study with nitrogen-13 ammonia and positron emission tomography. Journal of the American College of Cardiology, 17, 879-886.

5. Cannon, R. O., Dilsizian, V., O'Gara, P. T., et al. (1991). Myocardial metabolic, hemodynamic, and electrocardiographic significance of reversible thallium-201 abnormalities in hypertrophic cardiomyopathy. Circulation, 83, 1660-67.

6. Cannon, R. O., McIntosh, C. L., Schenke, W. H., et al. (1989). Effect of surgical reduction of left ventricular outflow tract obstruction on hemodynamics, coronary flow and myocardial metabolism in hypertrophic cardiomyopathy. Circulation, 79, 766-775.

7. Cecchi, F., Olivotto, I., Betocchi, S., et al. (2005). The Italian Registry for hypertrophic cardiomyopathy: A nationwide survey. American Heart Journal, 150(5), 947-954.

8. Cecchi, F., Olivotto, I., Gistri, R., et al. (2003). Coronary microvascular dysfunction and prognosis in hypertrophic cardiomyopathy. New England Journal of Medicine, 349, 1027-1035.

9. Cecchi, F., Olivotto, I., Montereggi, A., et al. (1995). Hypertrophic cardiomyopathy in Tuscany: Clinical course and outcome in an unselected regional population. Journal of the American College of Cardiology, 26, 1529-1536.
10. Cecchi, F., Olivotto, I., Nistri, S., Antoniucci, D., \& Yacoub, M. H. (2006). Midventricular obstruction and clinical decisionmaking in obstructive hypertrophic cardiomyopathy. Herz, 31(9), 871-876.

11. Coakley, E., Steinberg, D. H., Tibrewala, A., Asch, F., Pichard, A. D., Kent, K. M., et al. (2008). Effect of alcohol septal ablation in patients with hypertrophic cardiomyopathy on the electrocardiographic pattern. American Journal of Cardiology, 102(5), 621624.

12. Dilsizian, V. (2008). ${ }^{18}$ F-FDG uptake as a surrogate marker for antecedent ischemia. Journal of Nuclear Medicine, 49(12), 19091911.

13. Dou, K. F., Yang, M. F., Yang, Y. J., Jain, D., \& He, Z. X. (2008). Myocardial ${ }^{18}$ F-FDG uptake after exercise-induced myocardial ischemia in patients with coronary artery disease. Journal of Nuclear Medicine, 49(12), 1986-1991.

14. Elliott, P. M., Kaski, J. C., Prasad, K., Seo, H., et al. (1996). Chest pain during daily life in patients with hypertrophic cardiomyopathy: An ambulatory electrocardiographic study. European Heart Journal, 17, 1056-64.

15. Erdogan, D., Gullu, H., Caliskan, M., et al. (2007). Nebivolol improves coronary flow reserve in patients with idiopathic dilated cardiomyopathy. Heart, 93, 319-324.

16. Fighali, S., Krajcer, Z., Edelman, S., et al. (1987). Progression of hypertrophic cardiomyopathy into a hypokinetic left ventricle: Higher incidence in patients with midventricular obstruction. Journal of the American College of Radiology, 9(2), 288-294.

17. Gistri, R., Cecchi, F., Choudhury, L., Montereggi, A., Sorace, O., Salvadori, P. A., et al. (1994). Effect of verapamil on absolute myocardial blood flow in hypertrophic cardiomyopathy. American Journal of Cardiology, 74, 363-368.

18. Harris, K. M., Spirito, P., Maron, M. S., et al. (2006). Prevalence, clinical profile and significance of left ventricular remodeling in the end-stage phase of hypertrophic cardiomyopathy. Circulation, 114, 216-225.

19. Johansson, B., Mörner, S., Waldenström, A., \& Stål, P. (2008). Myocardial capillary supply is limited in hypertrophic cardiomyopathy: A morphological analysis. International Journal of Cardiology, 126(2), 252-257.

20. Kofflard, M. J., Michels, M., Krams, R., Kliffen, M., Geleijnse, M. L., Ten Cate, F. J., et al. (2007). Coronary flow reserve in hypertrophic cardiomyopathy: Relation with microvascular dysfunction and pathophysiological characteristics. Netherlands Heart Journal, 15(6), 209-215.

21. Krams, R., Kofflard, M. J., Duncker, D. J., Von Birgelen, C., Carlier, S., Kliffen, M., et al. (1998). Decreased coronary flow reserve in hypertrophic cardiomyopathy is related to remodeling of the coronary microcirculation. Circulation, 97(3), 230-233.

22. Lazzeroni, E., Picano, E., Morozzi, L., Maurizio, A. R., Palma, G., Ceriati, R., et al. (1997). Dipyridamole-induced ischemia as a prognostic marker of future adverse cardiac events in adult patients with hypertrophic cardiomyopathy. Echo Persantine Italian Cooperative (EPIC) Study Group, Subproject Hypertrophic Cardiomyopathy. Circulation, 96, 4268-4272.

23. Lorenzoni, R., Gistri, R., Cecchi, F., Olivotto, I., Chiriatti, G., Elliott, P., et al. (1997). Syncope and ventricular arrhythmias in hypertrophic cardiomyopathy are not related to the derangement of coronary microvascular function. European Heart Journal, 18, 1946-1950.

24. Losi, M. A., Betocchi, S., Aversa, M., Lombardi, R., Miranda, M., Cacace, A., et al. (2003). Dobutamine stress echocardiography in hypertrophic cardiomyopathy. Cardiology, 100, 93-100.

25. Maron, B. J. (2002). Hypertrophic cardiomyopathy: A systematic review. JAMA, 287, 1308-1320.

26. Maron, B. J., Epstein, S. E., \& Roberts, W. C. (1979). Hypertrophic cardiomyopathy and transmural myocardial infarc- 
tion without significant atherosclerosis of the extramural coronary arteries. American Journal of Cardiology, 43, 1086-1102.

27. Maron, B. J., McKenna, W. J., Danielson, G. K., Kappenberger, L. J., Kuhn, H. J., Seidman, C. E., et al. (2003). American College of Cardiology/European Society of Cardiology Clinical Expert Consensus Document on Hypertrophic Cardiomyopathy. A report of the American College of Cardiology Foundation Task Force on Clinical Expert Consensus Documents and the European Society of Cardiology Committee for Practice Guidelines. European Heart Journal, 24(21), 1965-1991.

28. Maron, M. S., Olivotto, I., Betocchi, S., et al. (2003). Effect of left ventricular outflow tract obstruction on clinical outcome in hypertrophic cardiomyopathy. New England Journal of Medicine, 348, 295-303.

29. Maron, M. S., Olivotto, I., Maron, B. J., Prasad, S. K., Cecchi, F., Udelson, J. E., et al. (2009). The case for myocardial ischemia in hypertrophic cardiomyopathy. Journal of the American College of Cardiology, 54(9), 866-875.

30. Maron, B. J., Olivotto, I., Spirito, P., et al. (2000). Epidemiology of hypertrophic cardiomyopathy-related death: Revisited in a large non-referral-based patient population. Circulation, 102, 858-864.

31. Maron, M. S., Olivotto, I., Zenovich, A. G., Link, M. S., Pandian, N. G., Kuvin, J. T., et al. (2006). Hypertrophic cardiomyopathy is predominantly a disease of left ventricular outflow tract obstruction. Circulation, 114, 2232-2239.

32. Maron, B. J., \& Spirito, P. (1998). Implications of left ventricular remodeling in hypertrophic cardiomyopathy. American Journal of Cardiology, 81, 1339-1344.

33. Maron, B. J., Wolfson, J. K., Epstein, S. E., et al. (1986). Intramural ("small vessel") coronary artery disease in hypertrophic cardiomyopathy. Journal of the American College of Cardiology, 8, 545-557.

34. Miyai, N., Kawasaki, T., Taniguchi, T., Kamitani, T., Kawasaki, S., \& Sugihara, H. (2005). Exercise-induced ST-segment depression and myocardial ischemia in patients with hypertrophic cardiomyopathy: Myocardial scintigraphic study. Journal of Cardiology, 46(4), 141-147.

35. Moon, J. C., McKenna, W. J., McCrohon, J. A., Elliott, P. M., Smith, G. C., \& Pennell, D. J. (2003). Toward clinic risk assessment in hypertrophic cardiomyopathy with gadolinium cardiovascular magnetic resonance. Journal of the American College of Cardiology, 41, 1561-1567.

36. Mourad, J. J., Hanon, O., Deverre, J. R., et al. (2003). Improvement of impaired coronary vasodilator reserve in hypertensive patients by low-dose ACE inhibitor/diuretic therapy: A pilot PET study. Journal of the Renin-Angiotensin-Aldosterone System, 4, 94-95.

37. Nikolaidis, L. A., Doverspike, A., Huerbin, R., Hentosz, T., \& Shannon, R. P. (2002). Angiotensin-converting enzyme inhibitors improve coronary flow reserve in dilated cardiomyopathy by a bradykinin-mediated, nitric oxide-dependent mechanism. Circulation, 105, 2785-2790.

38. Olivotto, I., Cecchi, F., Casey, S. A., Dolara, A., Traverse, J. H., \& Maron, B. J. (2001). Impact of atrial fibrillation on the clinical course of hypertrophic cardiomyopathy. Circulation, 104, 25172524.

39. Olivotto, I., Cecchi, F., Gistri, R., Lorenzoni, R., Chiriatti, G., Vargiu, D., et al. (2006). Relevance of coronary microvascular flow impairment to long-term remodeling and systolic dysfunction in hypertrophic cardiomyopathy. Journal of the American College of Cardiology, 47, 1043-1048.

40. Ommen, S. R., Maron, B. J., Olivotto, I., et al. (2005). Long-term effects of surgical septal myectomy on survival of patients with obstructive hypertrophic cardiomyopathy. Journal of the American College of Cardiology, 46, 470-476.

41. Parodi, O., Neglia, D., Palombo, C., Sambuceti, G., Giorgetti, A., Marabotti, C., et al. (1997). Comparative effects of enalapril and verapamil on myocardial blood flow in systemic hypertension. Circulation, 96, 864-873.

42. Pasternac, A., Noble, J., Streulens, Y., et al. (1982). Pathophysiology of chest pain in patients with cardiomyopathies and normal coronary arteries. Circulation, 62, 778-789.

43. Petersen, S. E., Jerosch-Herold, M., Hudsmith, L. E., Robson, M. D., Francis, J. M., Doll, H. A., et al. (2007). Evidence for microvascular dysfunction in hypertrophic cardiomyopathy: New insights from multiparametric magnetic resonance imaging. Circulation, 115, 2418-2425.

44. Rosing, D. R., Idanpaan-Heikkila, U., Maron, B. J., Bonow, R. O., \& Epstein, S. E. (1985). Use of calcium-channel blocking drugs in hypertrophic cardiomyopathy. American Journal of Cardiology, $55,185 \mathrm{~B}-195 \mathrm{~B}$.

45. Sciagrà, R., Sotgia, B., Olivotto, I., Cecchi, F., et al. (2009). Relationship between atrial fibrillation and blunted hyperemic myocardial blood flow in patients with hypertrophic cardiomyopathy. Journal of Nuclear Cardiology, 16, 92-96.

46. Sotgia, B., Sciagrà, R., Olivotto, I., Casolo, G., Rega, L., Betti, I., et al. (2008). Spatial relationship between coronary microvascular dysfunction and delayed contrast enhancement in patients with hypertrophic cardiomyopathy. Journal of Nuclear Medicine, 49, 1090-1096.

47. Stafford, W. J., Trohman, R. G., Bilsker, M., et al. (1986). Cardiac arrest in an adolescent with atrial fibrillation and hypertrophic cardiomyopathy. Journal of the American College of Cardiology, 7, 701-704

48. Wigle, E. D., Rakowski, H., Kimball, B. P., et al. (1995). Hypertrophic cardiomyopathy: Clinical spectrum and treatment. Circulation, 92, 1680-1692. 